\title{
Strong Fubini properties for measure and category
}

\author{
by
}

\author{
Krzysztof Ciesielski (Morgantown, WV) and \\ Miklós Laczkovich (Budapest and London)
}

Abstract. Let (FP) abbreviate the statement that

$$
\int_{0}^{1}\left(\int_{0}^{1} f d y\right) d x=\int_{0}^{1}\left(\int_{0}^{1} f d x\right) d y
$$

holds for every bounded function $f:[0,1]^{2} \rightarrow \mathbb{R}$ whenever each of the integrals involved exists. We shall denote by (SFP) the statement that the equality above holds for every bounded function $f:[0,1]^{2} \rightarrow \mathbb{R}$ having measurable vertical and horizontal sections. It follows from well-known results that both of (FP) and (SFP) are independent of the axioms of ZFC. We investigate the logical connections of these statements with several other strong Fubini type properties of the ideal of null sets. In particular, we establish the equivalence of (SFP) to the nonexistence of certain sets with paradoxical properties, a phenomenon that was already known for (FP). We also give the category analogues of these statements and, whenever possible, we try to put the statements in a setting of general ideals as initiated by Recław and Zakrzewski.

1. Introduction. In this paper we investigate the equality

$$
\int_{0}^{1}\left(\int_{0}^{1} f d y\right) d x=\int_{0}^{1}\left(\int_{0}^{1} f d x\right) d y
$$

under different conditions on the function $f:[0,1]^{2} \rightarrow \mathbb{R}$. It was already known to Cauchy that (1) holds for any continuous function $f:[0,1]^{2} \rightarrow \mathbb{R}$. Cauchy also knew (see [1]) that this is false when we allow just one point of discontinuity for $f$ : if $f(x, y)=\left(x^{2}-y^{2}\right) /\left(x^{2}+y^{2}\right)^{2}$ then the two sides of (1) equal $\pi / 4$ and $-\pi / 4$, respectively. (Another simple example is $f(x, y)=$ $(x-y) /(x+y)^{3}$, where the corresponding values are $-1 / 2$ and $1 / 2$.) However,

2000 Mathematics Subject Classification: 03E35, 26B20, 28A05.

This project has been funded in part by the National Academy of Sciences and under the Collaboration in Basic Science and Engineering Program supported by Contract No. INT-0002341 from the National Science Foundation. In addition, the first author was also partially supported by 2002/03 West Virginia University Senate Research Grant while the second author was partially supported by the Hungarian National Foundation for Scientific Research Grant No. T032042. 
it was proved in 1883 by du Bois-Reymond [4] that if $f$ is Riemann integrable on $[0,1]^{2}$ then both of the integrals $\int_{0}^{1}\left(\bar{\int}_{0}^{1} f d y\right) d x$ and $\int_{0}^{1}\left(\int_{0}^{1} f d x\right) d y$ are equal to the double integral $\iint_{[0,1]^{2}} f(x, y) d x d y$, where $\bar{\int}$ denotes the Darboux upper integral. In particular, if $f$ is Riemann integrable on $[0,1]^{2}$ and the integrals $\int_{0}^{1} f_{x} d y$ and $\int_{0}^{1} f^{y} d x$ exist for every $x, y \in[0,1]$ then (1) holds. Here $f_{x}, f^{y}:[0,1] \rightarrow \mathbb{R}$ are the sections of $f$, that is, $f_{x}(y)=f(x, y)=f^{y}(x)$ for every $x, y \in[0,1]$.

The most important theorem on (1) is that of Fubini [8] $\left({ }^{1}\right)$ stating that (1) holds provided that $f$ is summable on $[0,1] \times[0,1]$.

The first theorem stating (1) without assuming the measurability of $f$ was proved in 1911 by L. Lichtenstein [20] $\left({ }^{2}\right)$ who showed that (1) holds for every bounded function $f$ for which $f^{y}$ is Riemann integrable for every $y \in[0,1]$, and $f_{x}$ is measurable for every $x \in[0,1]$.

Motivated by Lichtenstein's result Sierpiński [22] proved that, under the continuum hypothesis $(\mathrm{CH})$, there exists a function $f:[0,1]^{2} \rightarrow\{0,1\}$ for which $\int_{0}^{1} f^{y} d x=0$ and $\int_{0}^{1} f_{x} d y=1$ for every $x, y \in[0,1]$. In particular, the iterated integrals for this function exist but are not equal, and so (1) fails. Actually, Sierpiński proves the following statement under $\mathrm{CH}$ :

$S^{2}$ : there exists a set $H \subset[0,1]^{2}$ such that the horizontal sections of $H$ are of measure zero and the vertical sections of $H$ are of full measure. That is, $\lambda\left(H^{y}\right)=\lambda\left([0,1] \backslash H_{x}\right)=0$ for every $x, y \in[0,1]$, where $\lambda$ is the Lebesgue measure, $H^{y}=\{x:\langle x, y\rangle \in H\}$ and $H_{x}=\{y:\langle x, y\rangle \in H\}$.

Then Sierpiński notes that the characteristic function of $H$ has the properties described above. This example shows that in the theorems of Fubini and Lichtenstein we cannot omit the conditions that $f$ is summable or that the sections $f^{y}$ are Riemann integrable.

Can Sierpiński's example be constructed in ZFC? The negative answer was given in 1980 by Friedman [7], who proved that there are models of ZFC in which the following statement, which we call the Fubini property, holds:

(FP) If $f:[0,1]^{2} \rightarrow \mathbb{R}$ is a bounded function such that $f_{x}$ and $f^{y}$ are measurable for every $x, y \in[0,1]$ and the mappings $[0,1] \ni x \mapsto$ $\int_{0}^{1} f(x, y) d y$ and $[0,1] \ni y \mapsto \int_{0}^{1} f(x, y) d x$ are measurable then (1) holds.

Later it was shown independently by Laczkovich [16] and Freiling [5] that $(\mathrm{FP})$ is, in fact, equivalent to the negation of the statement $S^{2}$. Now it is

$\left({ }^{1}\right)$ According to $[9$, p. 161] Fubini's proof was defective. Later different proofs were provided by Hobson [10] and de la Vallée-Poussin [3].

$\left({ }^{2}\right)$ Note that Sierpiński in [22] incorrectly refers this result to another paper of Lichtenstein of 1910. We like to thank J. Trzeciak for helping us to spot this error. 
easy to see that $S^{2}$ implies the inequality $\operatorname{cov}(\mathcal{N}) \leq \operatorname{non}(\mathcal{N})$. Here $\operatorname{cov}(\mathcal{N})$ denotes the minimum cardinality of a system of null sets that can cover $\mathbb{R}$ and $\operatorname{non}(\mathcal{N})$ is the minimum cardinality of a set of positive outer measure. It was shown by Kunen [14] that the inequality $\operatorname{cov}(\mathcal{N})>\operatorname{non}(\mathcal{N})$ is consistent with ZFC. Since this inequality implies $\neg S^{2}$, which, in turn, is equivalent to (FP), we find that the consistency of (FP) also follows from Kunen's theorem.

We may also ask whether or not the following stronger version of (FP), which we call the strong Fubini property, is consistent with ZFC:

(SFP) If $f:[0,1]^{2} \rightarrow \mathbb{R}$ is a bounded function such that $f_{x}$ and $f^{y}$ are measurable for every $x, y \in[0,1]$ then the mappings $[0,1] \ni x \mapsto$ $\int_{0}^{1} f(x, y) d y$ and $[0,1] \ni y \mapsto \int_{0}^{1} f(x, y) d x$ are measurable and (1) holds.

Although we did not find in the literature (SFP) in its specific form, it follows easily from the following strong approximation property (for measure):

$(\mathrm{SAP}) \quad$ If $f:[0,1]^{2} \rightarrow \mathbb{R}$ is such that $f_{x}$ and $f^{y}$ are measurable for every $x, y \in[0,1]$ then there is a measurable function $F:[0,1]^{2} \rightarrow \mathbb{R}$ such that $f_{x}=F_{x}$ a.e. and $f^{y}=F^{y}$ a.e. for every $x, y \in[0,1]$.

In [21, p. 139], the theorem stating the consistency of (SAP) is attributed to H. Woodin. The first published proof can be found in [21, Theorem 2.17(ii)]. Therefore (SAP) and, consequently, (SFP) are both consistent with ZFC.

As we shall see later, the statements (SAP) and (SFP) are, in fact, equivalent. One of the goals of this paper is to present several other statements equivalent to (SFP), including one that states the nonexistence of certain sets with paradoxical properties, analogously to the equivalence (FP) $\Leftrightarrow \neg S^{2}$. All these statements can be expressed in a setting of general ideals on Polish spaces; we will do this in the next section.

2. Kuratowski-Ulam type theorems for ideals. We use standard set-theoretic terminology as in [2].

Let $X$ be a Polish space and let $\mathcal{I}$ be an ideal in $X$. We shall denote by $\mathcal{B}_{\mathcal{I}}$ the $\sigma$-algebra generated by $\mathcal{I}$ and by the Borel subsets $\mathcal{B}$ of $X$. The ideals that are the most interesting for us are the $\sigma$-ideals $\mathcal{N}$ of Lebesgue measure zero subsets of $\mathbb{R}$ and $\mathcal{M}$ of meager subsets of $\mathbb{R}$. Then $\mathcal{B}_{\mathcal{N}}$ and $\mathcal{B}_{\mathcal{M}}$ are the families of measurable sets and of sets with the Baire property, respectively. The sections of a set $A \subset X^{2}$ are defined by

$$
A_{x}=\{y \in X:\langle x, y\rangle \in A\} \quad \text { and } \quad A^{y}=\{x \in X:\langle x, y\rangle \in A\}
$$

for every $x, y \in X$. 
Recall that the Kuratowski-Ulam theorem says that for every $A \subset \mathbb{R}^{2}$ with the Baire property, if $A_{x} \in \mathcal{M}$ for every $x \in \mathbb{R}$ then $\left\{y: A^{y} \notin \mathcal{M}\right\} \in \mathcal{M}$. Thus the following Kuratowski-Ulam property of an arbitrary ideal $\mathcal{I}$ is a stronger version of the theorem, when considered with $\mathcal{I}=\mathcal{M}$.

(KU) If $A \subset X^{2}$ is such that $A_{x} \in \mathcal{I}$ and $A^{y} \in \mathcal{B}_{\mathcal{I}}$ for every $x, y \in X$ and $\left\{y: A^{y} \notin \mathcal{I}\right\} \in \mathcal{B}_{\mathcal{I}}$, then $\left\{y: A^{y} \notin \mathcal{I}\right\} \in \mathcal{I}$.

We will also consider the following two strong Kuratowski-Ulam properties: (SKU) If $A \subset X^{2}$ is such that $A_{x} \in \mathcal{I}$ and $A^{y} \in \mathcal{B}_{\mathcal{I}}$ for every $x, y \in X$, then $\left\{y: A^{y} \notin \mathcal{I}\right\} \in \mathcal{I}$.

(SKU*) If $A \subset X^{2}$ is such that $A_{x} \in \mathcal{B}_{\mathcal{I}}$ and $A^{y} \in \mathcal{B}_{\mathcal{I}}$ for every $x, y \in X$, then $\left\{y: A^{y} \notin \mathcal{I}\right\} \in \mathcal{B}_{\mathcal{I}}$.

Note that Recław and Zakrzewski in [21] refer to (SKU) as "strong Fubini property" and denote it as (SFP). However, we prefer to reserve the word "Fubini" for the properties that refer explicitly to the properties of integrals.

Our first aim is to establish the logical connections between these three statements and the nonexistence of sets having some paradoxical properties with respect to the ideal $\mathcal{I}$. We shall say that a set $H \subset A \times B$ is a $0-1$ set $\left(\right.$ in $A \times B$ ) provided $H^{y} \in \mathcal{I}$ for every $y \in B$ and $B \backslash H_{x} \in \mathcal{I}$ for every $x \in A$. Note that

(2) if there is a $0-1$ set in $A \times B$ then there is also one in $B \times A$.

Indeed, if $H$ is a $0-1$ set in $A \times B$ then $\{\langle x, y\rangle \in B \times A$ : $\langle y, x\rangle \notin H\}$ is a 0-1 set in $B \times A$.

We shall use the notation $A^{\mathrm{c}}=X \backslash A$ for every $A \subset X$. We will also use the following propositions concerning the existence of various $0-1$ sets.

$S_{\mathcal{I}}^{2}$ : There exists a $0-1$ set in $X \times X$.

$S_{\mathcal{I}}^{2 w}: \quad$ There exists a set $A \subset X$ not in $\mathcal{I}$ such that $A \times X$ contains a $0-1$ set.

$E_{\mathcal{I}}: \quad$ There exist sets $A, B \subset X$ not in $\mathcal{I}$ such that both $A \times B$ and $A^{\mathrm{c}} \times B^{\mathrm{c}}$ contain $0-1$ sets.

To state the next theorem, we also need the following classical cardinal numbers connected to $\mathcal{I}$ :

$$
\begin{aligned}
\operatorname{add}(\mathcal{I}) & =\min \{|\mathcal{F}|: \mathcal{F} \subset \mathcal{I} \& \bigcup \mathcal{F} \notin \mathcal{I}\} \\
\operatorname{cov}(\mathcal{I}) & =\min \{|\mathcal{F}|: \mathcal{F} \subset \mathcal{I} \& \bigcup \mathcal{F}=X\}, \\
\operatorname{non}(\mathcal{I}) & =\min \{|A|: A \subset X \& A \notin \mathcal{I}\} \\
\operatorname{shr}(\mathcal{I}) & =\min \left\{\kappa: \forall A \subset X\left(A \notin \mathcal{I} \Rightarrow[A]^{\kappa} \backslash \mathcal{I} \neq \emptyset\right)\right\} .
\end{aligned}
$$

In other words, $\operatorname{shr}(\mathcal{I})$ is the smallest cardinal $\kappa$ such that any subset $A$ of $X$ not belonging to $\mathcal{I}$ contains a subset $B$ such that $|B| \leq \kappa$ and $B \notin \mathcal{I}$. Clearly $\operatorname{non}(\mathcal{I}) \leq \operatorname{shr}(\mathcal{I})$. 
Theorem 1. Let $\mathcal{I}$ be an ideal in an uncountable Polish space $X$. Then we have the following implications:

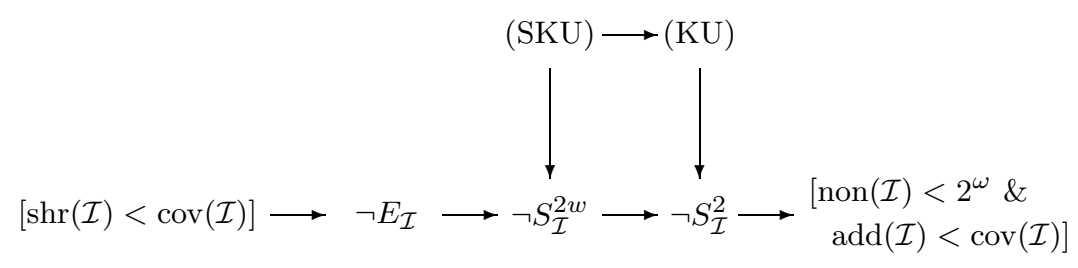

Moreover, if $\mathcal{I}$ satisfies the following condition: every set $B \in \mathcal{B}_{\mathcal{I}} \backslash \mathcal{I}$ contains a subset $S \notin \mathcal{B}_{\mathcal{I}}$,

then the chart can be expanded as

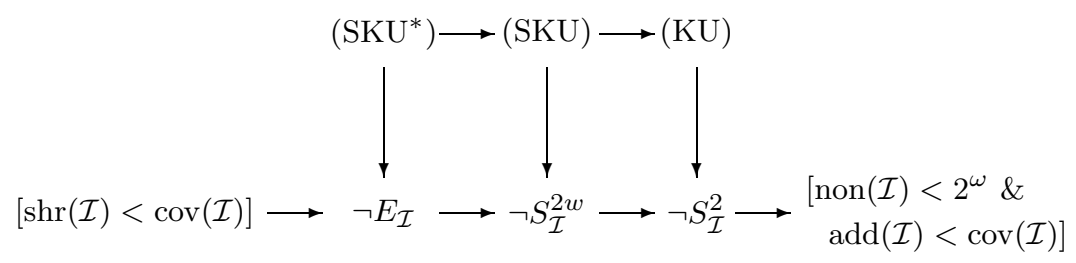

Proof. (SKU*) $\Rightarrow$ (SKU): The argument for this implication is closely related to that for [21, Theorem 2.11]. Let $A \subset X^{2}$ and suppose that $A_{x} \in \mathcal{I}$ and $A^{y} \in \mathcal{B}_{\mathcal{I}}$ for every $x, y \in X$. By $\left(\mathrm{SKU}^{*}\right)$ we have $C:=\left\{y: A^{y} \notin \mathcal{I}\right\} \in \mathcal{B}_{\mathcal{I}}$. If $C \notin \mathcal{I}$ then, by $(*)$, there is a subset $D \subset C$ such that $D \notin \mathcal{B}_{\mathcal{I}}$. Put

$$
H=A \cap(X \times D) .
$$

Then $H^{y} \in \mathcal{B}_{\mathcal{I}}$ for every $y \in X$, since $y \in D$ implies $H^{y}=A^{y}$ and $y \notin D$ implies $H^{y}=\emptyset$. Since $H \subset A$, we also have $H_{x} \in \mathcal{I}$ for every $x \in X$. Applying $\left(\mathrm{SKU}^{*}\right)$ again we find that

$$
\left\{y: H^{y} \notin \mathcal{I}\right\}=D \in \mathcal{B}_{\mathcal{I}},
$$

which is impossible.

$\left(\mathrm{SKU}^{*}\right) \Rightarrow \neg E_{\mathcal{I}}$ : Suppose that $E_{\mathcal{I}}$ holds. Then there are sets $A, B \subset X$, $H \subset A \times B$, and $K \subset A^{\mathrm{c}} \times B^{\mathrm{c}}$ such that $A \notin \mathcal{I}, B \notin \mathcal{I}, H$ is a $0-1$ set in $A \times B$, and $K$ is a $0-1$ set in $A^{\mathrm{c}} \times B^{\mathrm{c}}$.

First assume that $A, B \in \mathcal{B}_{\mathcal{I}}$. Then $H_{x}, H^{y} \in \mathcal{B}_{\mathcal{I}}$ for every $x, y \in X$. By $(*)$, there is a set $D \subset B$ such that $D \notin \mathcal{B}_{\mathcal{I}}$. Putting $E=H \cup(A \times D)$ we have $E_{x}, E^{y} \in \mathcal{B}_{\mathcal{I}}$ for every $x, y \in X$, and $\left\{y: E^{y} \notin \mathcal{I}\right\}=D \notin \mathcal{B}_{\mathcal{I}}$. This, however, contradicts $\left(\mathrm{SKU}^{*}\right)$.

Therefore we may assume that at least one of $A$ and $B$ does not belong to $\mathcal{B}_{\mathcal{I}}$. By symmetry we may suppose $B \notin \mathcal{B}_{\mathcal{I}}$. Put

$$
M=H \cup\left(\left(A^{\mathrm{c}} \times B^{\mathrm{c}}\right) \backslash K\right) \cup\left(A \times B^{\mathrm{c}}\right)
$$


and notice that $M$ refutes (SKU*). Indeed, to see that the sections of $M$ belong to $\mathcal{B}_{\mathcal{I}}$ note that if $y \in B$ then $M^{y}=H^{y} \in \mathcal{I}$ and for $y \in B^{\mathrm{c}}$ we have $X \backslash M^{y}=K^{y} \in \mathcal{I}$. Similarly, if $x \in A$ then $X \backslash M_{x}=B \backslash H_{x} \in \mathcal{I}$ and for $x \in A^{\mathrm{c}}$ we have $M_{x}=A^{\mathrm{c}} \backslash K_{x} \in \mathcal{I}$. However, $\left\{y: M^{y} \notin \mathcal{I}\right\}=B \notin \mathcal{B}_{\mathcal{I}}$ contradicts $\left(\mathrm{SKU}^{*}\right)$.

$(\mathrm{SKU}) \Rightarrow(\mathrm{KU})$ : This implication is immediate from the definitions.

All the remaining implications will be proved by contraposition.

(SKU) $\Rightarrow \neg S_{\mathcal{I}}^{2 w}$ : Suppose that $S_{\mathcal{I}}^{2 w}$ holds. Then, by (2), there are sets $A \subset X$ and $H \subset X \times A$ such that $A \notin \mathcal{I}$ and $H$ is a $0-1$ set in $X \times A$. Let $K=(X \times A) \backslash H$. Then $K_{x} \in \mathcal{I}$ and $K^{y} \in \mathcal{B}_{\mathcal{I}}$ for every $x, y \in X$ while $\left\{y: K^{y} \notin \mathcal{I}\right\}=A \notin \mathcal{I}$, which contradicts (SKU).

$(\mathrm{KU}) \Rightarrow \neg S_{\mathcal{I}}^{2}$ : If $S_{\mathcal{I}}^{2}$ holds then there is a $0-1$ set in $X^{2}$, which clearly contradicts (KU).

$\neg S_{\mathcal{I}}^{2} \Rightarrow\left[\operatorname{non}(\mathcal{I})<2^{\omega} \& \operatorname{add}(\mathcal{I})<\operatorname{cov}(\mathcal{I})\right]:$ A proof that there is a $0-1$ set if either $\operatorname{non}(\mathcal{I})=2^{\omega}$ or $\operatorname{add}(\mathcal{I})=\operatorname{cov}(\mathcal{I})$ can be found, for example, in $[17$, Theorem 2]. (Note that the proof given in [17] is valid for every ideal.)

$\neg S_{\mathcal{I}}^{2 w} \Rightarrow \neg S_{\mathcal{I}}^{w}$ : The implication $S_{\mathcal{I}}^{2} \Rightarrow S_{\mathcal{I}}^{2 w}$ is obvious.

$\neg E_{\mathcal{I}} \Rightarrow \neg S_{\mathcal{I}}^{2 w}$ : Assume that $S_{\mathcal{I}}^{2 w}$ holds. Then there are sets $A \subset X$ and $H \subset A \times X$ such that $A \notin \mathcal{I}$ and $H$ is a 0-1 set in $A \times X$. If $A^{\mathrm{c}} \in \mathcal{I}$ then $E_{\mathcal{I}}$ is satisfied with $B=A$. If $A^{\mathrm{c}} \notin \mathcal{I}$ then $E_{\mathcal{I}}$ is satisfied with $B=A^{\mathrm{c}}$, since $H \cap\left(A \times A^{\mathrm{c}}\right)$ is a $0-1$ set in $A \times A^{\mathrm{c}}$, and the existence of a $0-1$ set in $A^{\mathrm{c}} \times A$ follows from (2).

$[\operatorname{shr}(\mathcal{I})<\operatorname{cov}(\mathcal{I})] \Rightarrow \neg E_{\mathcal{I}}$ : For the proof of $E_{\mathcal{I}} \Rightarrow[\operatorname{shr}(\mathcal{I}) \geq \operatorname{cov}(\mathcal{I})]$ we shall need the following lemma, which is implicit in [5, p. 193] and [21, Lemma 2.7]. For the sake of completeness we give the simple proof. In the lemma we use the notation $\mathcal{I} \mid A$ to denote the ideal $\{B \in \mathcal{I}: B \subset A\}$.

Lemma 2. If $A, B \subset X, A, B \notin \mathcal{I}$, and there is a 0-1 set in $A \times B$ then $\operatorname{cov}(\mathcal{I} \mid A) \leq \operatorname{non}(\mathcal{I} \mid B)$.

Proof. Let $H$ be a $0-1$ set in $A \times B$. Then $H^{y} \in \mathcal{I}$ for every $y \in B$ and $B \backslash H_{x} \in \mathcal{I}$ for every $x \in A$. If $D \subset B,|D|=\operatorname{non}(\mathcal{I} \mid B)$, and $D \notin \mathcal{I}$, then $A=\bigcup_{y \in D} H^{y}$, since $x \in A \backslash \bigcup_{y \in D} H^{y}$ would imply $H_{x} \cap D=\emptyset$, contradicting $B \backslash H_{x} \in \mathcal{I}$. Thus $\operatorname{cov}(\mathcal{I} \mid A) \leq|D|=\operatorname{non}(\mathcal{I} \mid B)$.

Now we turn to the proof of $E_{\mathcal{I}} \Rightarrow[\operatorname{shr}(\mathcal{I}) \geq \operatorname{cov}(\mathcal{I})]$. By $E_{\mathcal{I}}$, there are sets $A, B \notin \mathcal{I}$ such that both of $A \times B$ and $A^{\mathrm{c}} \times B^{\mathrm{c}}$ contain 0-1 sets. If 
$A^{\mathrm{c}} \notin \mathcal{I}$ and $B^{\mathrm{c}} \notin \mathcal{I}$ then Lemma 2 gives $\operatorname{cov}(\mathcal{I} \mid A) \leq \operatorname{non}(\mathcal{I} \mid B) \leq \operatorname{shr}(\mathcal{I})$ and $\operatorname{cov}\left(\mathcal{I} \mid A^{\mathrm{c}}\right) \leq \operatorname{non}\left(\mathcal{I} \mid B^{\mathrm{c}}\right) \leq \operatorname{shr}(\mathcal{I})$, and hence

$$
\operatorname{cov}(\mathcal{I}) \leq \max \left\{\operatorname{cov}(\mathcal{I} \mid A), \operatorname{cov}\left(\mathcal{I} \mid A^{\mathrm{c}}\right)\right\} \leq \operatorname{shr}(\mathcal{I}) .
$$

On the other hand, if $A^{\mathrm{c}} \in \mathcal{I}$ then there is a 0-1 set in $X \times B$ and thus $\operatorname{cov}(\mathcal{I}) \leq \operatorname{non}(\mathcal{I} \mid B) \leq \operatorname{shr}(\mathcal{I})$. We have the same conclusion if $B^{\mathrm{c}} \in \mathcal{I}$.

For a semigroup $G$ of Borel functions from $X$ to $X$ and an ideal $\mathcal{I}$ on $X$ we say that $\mathcal{I}$ is $G$-invariant provided $g^{-1}(A) \in \mathcal{I}$ for every $g \in G$ and $A \in \mathcal{I}$; and $\mathcal{I}$ is $G$-ergodic when $X \backslash \bigcup_{g \in G} g^{-1}(A) \in \mathcal{I}$ for every $A \in \mathcal{B}_{\mathcal{I}} \backslash \mathcal{I}$. Clearly if $G$ is the group of rational translations in $\mathbb{R}$ then the ideals $\mathcal{N}$ and $\mathcal{M}$ are $G$-invariant and $G$-ergodic.

If we strengthen a bit the assumptions of the main part of Theorem 1 then we can get a few more implications.

Corollary 3. Let $\mathcal{I}$ be a $\sigma$-ideal in the Polish space $X$ and suppose that there is a countable semigroup $G$ of Borel functions from $X$ to $X$ such that $\mathcal{I}$ is $G$-invariant and $G$-ergodic. Then we have the following implications:

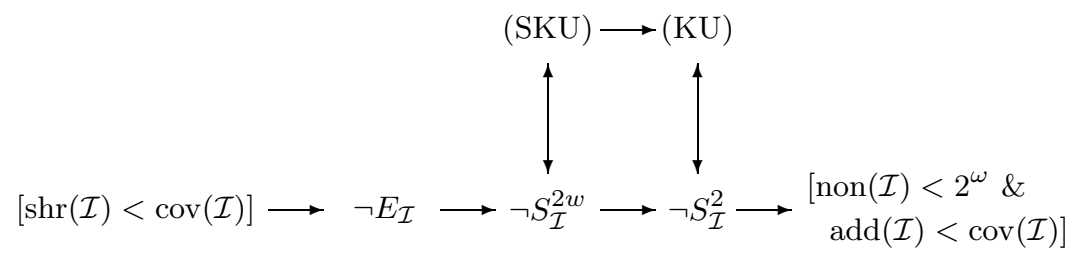

Proof. The equivalence (SKU) $\Leftrightarrow \neg S_{\mathcal{I}}^{2 w}$ is proved in [21, Lemma 2.8]. The same argument gives $\neg S_{\mathcal{I}}^{2} \Leftrightarrow(\mathrm{KU})$. The other implications follow from Theorem 1.

We do not know if the hypothesis above also implies $\left(\mathrm{SKU}^{*}\right) \Leftrightarrow \neg E_{\mathcal{I}}$ even in the presence of $(*)$. However, as we shall see later, this equivalence holds for $\mathcal{I}=\mathcal{N}$ and $\mathcal{I}=\mathcal{M}$.

The condition $(*)$ was only used in the proof of Theorem 1 in the implications $\left(\mathrm{SKU}^{*}\right) \Rightarrow \neg E_{\mathcal{I}}$ and $\left(\mathrm{SKU}^{*}\right) \Rightarrow$ (SKU). The following example shows that it is really needed in these arguments.

EXAMPLE 4. Let $X=\mathbb{R}$ and let $\mathcal{I}$ be a prime ideal containing all sets of cardinality less than $2^{\omega}$. For this ideal (SKU*) holds while all other statements of the diagram are false.

Proof. Since $\mathcal{I}$ is prime, we have $\mathcal{B}_{\mathcal{I}}=\mathcal{P}(\mathbb{R})$. Thus (SKU*) holds. On the other hand, clearly non $(\mathcal{I})=2^{\omega}$, so the statements of the diagram are false. 
We shall also investigate the following Borel approximation property: (BAP) if $A \subset X^{2}$ is such that $A_{x} \in \mathcal{B}_{\mathcal{I}}$ and $A^{y} \in \mathcal{B}_{\mathcal{I}}$ for every $x, y \in X$, then there is a Borel set $B \subset X^{2}$ such that $(A \triangle B)_{x} \in \mathcal{I}$ for $\mathcal{I}$-a.e. $x \in X$ and $(A \triangle B)^{y} \in \mathcal{I}$ for $\mathcal{I}$-a.e. $y \in X$.

We will leave the following simple fact without a proof.

FACT 5. If $\mathcal{I}$ is a $\sigma$-ideal on $X$ then $(\mathrm{BAP})$ is equivalent to the following statement:

- If $f: X^{2} \rightarrow \mathbb{R}$ is such that $f_{x}$ and $f^{y}$ are $\mathcal{B}_{\mathcal{I}}$-measurable for every $x, y \in X$ then there is a Borel function $g: X^{2} \rightarrow \mathbb{R}$ such that $f_{x}=g_{x}$ $\mathcal{I}$-a.e. and $f^{y}=g^{y} \mathcal{I}$-a.e. for $\mathcal{I}$-almost every $x, y \in X$.

Also, (BAP) is equivalent to (SAP) for $\mathcal{I}=\mathcal{N}$ and for $\mathcal{I}=\mathcal{M}$.

We state the equivalence to (SAP) only for the ideals $\mathcal{N}$ and $\mathcal{M}$ to avoid the problem of defining $\mathcal{B}_{\mathcal{I}}$-measurability of a function from $X^{2}$ into $\mathbb{R}$. (A generalization of (SAP) to the category case is natural.)

Notice that it would not be reasonable to require in (BAP) the existence of a Borel set $B$ such that $(A \triangle B)_{x} \in \mathcal{I}$ and $(A \triangle B)^{y} \in \mathcal{I}$ for every $x$ and $y$. In fact, this stronger form of (BAP) is satisfied neither by $\mathcal{N}$ nor by $\mathcal{M}$ as the following simple example shows.

Let $H$ be a non-Borel subset of the Cantor set, and put $A=H \times[0,1]$. Then $A_{x} \in \mathcal{B}_{\mathcal{I}}$ and $A^{y} \in \mathcal{B}_{\mathcal{I}}$ for every $x, y \in[0,1]$ for both $\mathcal{I}=\mathcal{N}$ and $\mathcal{I}=\mathcal{M}$. Suppose there is a Borel set $B \subset[0,1]^{2}$ such that $(A \triangle B)_{x} \in \mathcal{N}$ for every $x \in[0,1]$. Then $\left\{x \in[0,1]: B_{x} \notin \mathcal{N}\right\}=H$, contradicting the fact that this set must be Borel whenever $B$ is Borel. (See e.g. [12, (16.1) Theorem, p. 94].) The same argument works for $\mathcal{I}=\mathcal{M}$.

In what follows we will prove that (SAP) is equivalent to $\left(\mathrm{SKU}^{*}\right)$ for $\mathcal{I}=\mathcal{N}$ and $\mathcal{I}=\mathcal{M}$. We start in this direction by noticing the following simple fact, which will be left without a proof.

Proposition 6. Suppose that $\left\{y: A^{y} \notin \mathcal{I}\right\} \in \mathcal{B}_{\mathcal{I}}$ for every Borel set $A \subset X^{2}$. Then $(\mathrm{BAP}) \Rightarrow\left(\mathrm{SKU}^{*}\right)$.

Note that the assumption of Proposition 6 is not satisfied by every ideal. For example, if $\mathcal{I}$ equals the ideal of countable sets or of first category null sets then there is a Borel set $A \subset \mathbb{R}^{2}$ such that $\left\{y: A^{y} \notin \mathcal{I}\right\} \notin \mathcal{B}_{\mathcal{I}}$.

We do not know whether or not (BAP) implies (SKU*) for every ideal.

3. Strong Fubini properties for measure. It was proved by C. Freiling in [5] (see also Laczkovich [16]) that (FP) is equivalent to $\neg S_{\mathcal{N}}^{2}$. Our aim here is to give a similar characterization of (SFP), by showing that it is equivalent to $\neg E_{\mathcal{N}}$ as well as to several other strong Fubini type proper- 
ties, including (BAP) for $\mathcal{N}$. Recall that $\mathcal{B}_{\mathcal{N}}$ coincides with the $\sigma$-algebra of measurable subsets of $\mathbb{R}$.

Notice also that the chart implies that all the properties considered there are independent of the axioms of ZFC. Indeed, the relations $\operatorname{add}(\mathcal{N})=$ $\operatorname{cov}(\mathcal{N})=\operatorname{non}(\mathcal{N})=2^{\omega}$ and $\operatorname{shr}(\mathcal{N})<\operatorname{cov}(\mathcal{N})$ are both consistent with ZFC: the first is a consequence of Martin's Axiom, while the second holds in the random reals model. (For the proof of $\operatorname{shr}(\mathcal{N})<\operatorname{cov}(\mathcal{N})$ see [18] or [11]. This was certainly already known to Kunen [14].)

THEOREM 7. The following statements are equivalent to each other:

(i) If $f$ is a bounded function on $[0,1]^{2}$ such that the sections $f_{x}$ and $f^{y}$ are measurable for every $x, y \in[0,1]$ then the mapping $x \mapsto \int_{0}^{1} f(x, y) d y$ is measurable on $[0,1]$.

(ii) The strong Fubini property (SFP) holds.

(iii) $\mathcal{N}$ has property (SAP).

(iv) $\mathcal{N}$ has property (BAP).

(v) $\mathcal{N}$ has property (SKU*).

(vi) $E_{\mathcal{N}}$ is false.

In particular, for $\mathcal{I}=\mathcal{N}$ we have the following relations:

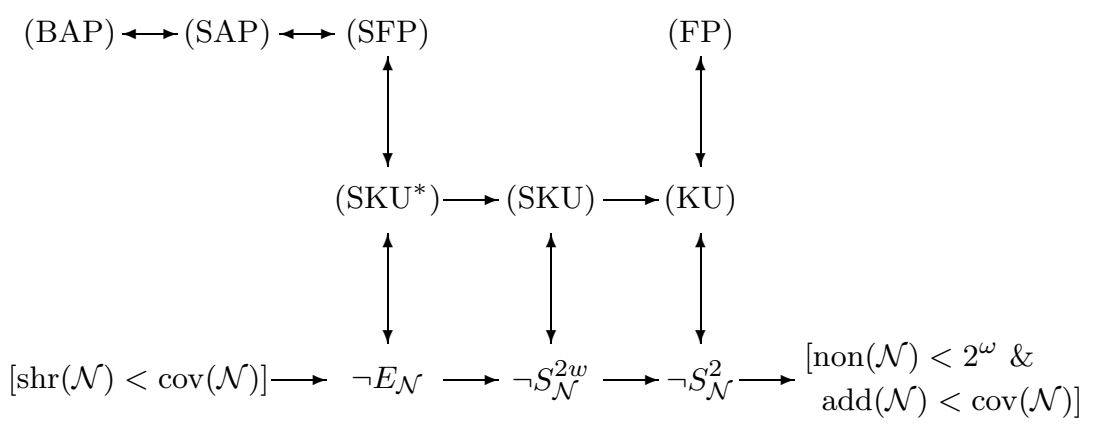

Proof. The implications in the chart follow from the main part of the theorem, from Freiling's result quoted above, as well as from Theorem 1 and Corollary 3.

In the course of the proof the sign $\int$ will abbreviate $\int_{0}^{1}$ unless another domain is indicated.

(i) $\Rightarrow\left(\right.$ ii): Let $f$ be a bounded function on $[0,1]^{2}$ such that the sections $f_{x}$ and $f^{y}$ are measurable for every $x, y \in[0,1]$. By (i), for every $x, y \in[0,1]$ the mappings $x \mapsto \int f(x, y) d y$ and $y \mapsto \int f(x, y) d x$ are measurable. We need to prove that the integrals $I_{1}=\int\left(\int f(x, y) d y\right) d x$ and $I_{2}=\int\left(\int f(x, y) d x\right) d y$ are equal.

By way of contradiction assume that $I_{1} \neq I_{2}$. Then, by Freiling's theorem [5], there exists a $0-1$ set $A \subset[0,1]^{2}$. After removing from $A$ some 
vertical sections we obtain a set $B$ such that $B^{y} \in \mathcal{N}$ for every $y$, and $\left\{x \in[0,1]:[0,1] \backslash B_{x} \in \mathcal{N}\right\}$ is not measurable. But then the characteristic function of $B$ refutes (i).

(ii) $\Rightarrow$ (iii): Suppose (SFP). Let $f:[0,1]^{2} \rightarrow \mathbb{R}$ be a function with measurable sections. We have to show that there is a measurable function $F:[0,1]^{2} \rightarrow \mathbb{R}$ such that $F_{x}=f_{x}$ a.e. for every $x \in[0,1]$ and $F^{y}=f^{y}$ a.e. for every $y \in[0,1]$. In the proof we may assume that $f$ is bounded. For $x, y \in[0,1]$ put $G(x, y)=\int_{0}^{y} f(x, t) d t$. Then $G$ is well defined and measurable. Indeed, by (SFP), $G^{y}$ is measurable for every $y \in[0,1]$. Also, $G_{x}$ is continuous for every $x \in[0,1]$. Thus, by Lebesgue's theorem [19], $G$ is measurable. Let $H=\frac{\partial}{\partial y} G$ where $\frac{\partial}{\partial y} G$ exists, and $H=0$ elsewhere. Then $H$ is measurable, and for every $x \in[0,1]$ we have $H_{x}(y)=f_{x}(y)$ for almost all $y \in[0,1]$. Then, by (SFP) and the Fubini theorem,

$$
\begin{aligned}
\int_{0}^{b}\left(\int_{0}^{a} f^{y}(x) d x\right) d y & =\int_{0}^{a}\left(\int_{0}^{b} f_{x}(y) d y\right) d x \\
& =\int_{0}^{a}\left(\int_{0}^{b} H_{x}(y) d y\right) d x \\
& =\int_{0}^{b}\left(\int_{0}^{a} H^{y}(x) d x\right) d y
\end{aligned}
$$

for every $a, b \in[0,1]$. Thus, for every $a \in[0,1]$ and almost every $y \in[0,1]$ we have $\int_{0}^{a} f^{y}(x) d x=\int_{0}^{a} H^{y}(x) d x$. So, for almost every $y \in[0,1]$ we have $\int_{0}^{a} f^{y}(x) d x=\int_{0}^{a} H^{y}(x) d x$ for every rational $a \in[0,1]$. Thus, by the continuity of the integral, for almost every $y \in[0,1]$ we have $\int_{0}^{a} f^{y}(x) d x=$ $\int_{0}^{a} H^{y}(x) d x$ for every $a \in[0,1]$. Then, for almost every $y \in[0,1]$, that is, for $y$ 's outside some null set $T \subset[0,1]$, we have $H^{y}(x)=f^{y}(x)$ for almost every $x \in[0,1]$. To get $F$ as desired define it to be equal to $f$ on $[0,1] \times T$ and to $H$ elsewhere.

(iii) $\Rightarrow$ (iv): This is obvious, and follows from Fact 5 .

(iv) $\Rightarrow(\mathrm{v})$ : If $A \subset[0,1]^{2}$ is Borel then the set $\left\{y \in[0,1]: A^{y} \notin \mathcal{N}\right\}$ is measurable by Fubini's theorem. Thus we may apply Proposition 6 .

$(\mathrm{v}) \Rightarrow(\mathrm{vi})$ : This was proved in Theorem 1 .

$(v i) \Rightarrow(i)$ : Suppose that (i) is false. Then there exists a bounded function $f:[0,1]^{2} \rightarrow \mathbb{R}$ such that the sections of $f$ are measurable, but the function $h(x)=\int f_{x} d y$, defined for $x \in[0,1]$, is not. Fix a real number $b$ such that the set $H=\{x \in[0,1]: h(x)<b\}$ is not measurable, and define $K=\{x \in[0,1]: h(x) \geq b\}$. Then $\lambda(H)+\lambda(K)>1$, where $\lambda$ stands for 
Lebesgue outer measure. Thus, there exists a real number $a<b$ such that $\lambda\left(H_{a}\right)+\lambda(K)>1$, where $H_{a}=\{x \in[0,1]: h(x) \leq a\}$.

Select measurable sets $M$ and $N$ such that $H_{a} \subset M, \lambda\left(H_{a}\right)=\lambda(M)$, $K \subset N$, and $\lambda(K)=\lambda(N)$. Then $\lambda(M)+\lambda(N)=\lambda\left(H_{a}\right)+\lambda(K)>1$, and thus $P=M \cap N$ is of positive measure. Clearly, $\lambda(P)=\lambda\left(H_{a} \cap P\right)=$ $\lambda(K \cap P)$. The measure space $\left\langle P, \frac{1}{\lambda(P)}(\lambda\lceil P)\rangle\right.$ is isomorphic to $\langle[0,1], \lambda\rangle$. Let $\phi$ be an isomorphism from $[0,1]$ onto $P$, and put $f_{1}(x, y)=f(\phi(x), y)$ for $x, y \in[0,1]$. Then the sections of $f_{1}$ are measurable and both of the sets $U=\left\{x \in[0,1]: \int f_{1}(x, y) d y \leq a\right\}$ and $V=\left\{x \in[0,1]: \int f_{1}(x, y) d y \geq b\right\}$ have outer measure 1 .

We shall denote by $\Omega$ the product space $[0,1]^{\omega}$ equipped with the product measure $\mu$ and define $\bar{\mu}(H)=\inf \{\mu(M): H \subset M$ and $M$ is $\mu$-measurable $\}$ for every $H \subset \Omega$. For $x \in[0,1]$ and $v=\left\langle v_{1}, v_{2}, \ldots\right\rangle \in \Omega$ let

$$
F(x, v)=\limsup _{n \rightarrow \infty} \frac{f_{1}\left(x, v_{1}\right)+\ldots+f_{1}\left(x, v_{n}\right)}{n} .
$$

By the strong law of large numbers for every $x \in[0,1]$ the equation $F(x, v)=$ $\int f_{1}(x, y) d y$ holds for $\mu$-a.e. $v \in \Omega$. Thus, $F_{x}$ is $\mu$-a.e. constant for every $x \in[0,1]$ since $F_{x}(v)=\int f_{1}(x, y) d y=F_{x}\left(v^{\prime}\right)$ for $\mu$-a.e. $v, v^{\prime} \in \Omega$. Moreover, for $x \in U$ we have $F(x, v) \leq a$ for $\mu$-a.e. $v \in \Omega$, and for $x \in V$ we have $F(x, v) \geq b$ for $\mu$-a.e. $v \in \Omega$. Also, $F^{v}(x)$ is measurable for every $v \in \Omega$, being the lim sup of measurable functions $\frac{1}{n}\left[\left(f_{1}\right)^{v_{1}}(x)+\ldots+\left(f_{1}\right)^{v_{n}}(x)\right]$. Now put

$$
G(u, v)=\limsup _{n \rightarrow \infty} \frac{F\left(u_{1}, v\right)+\ldots+F\left(u_{n}, v\right)}{n}
$$

for every $u, v \in \Omega$. Then, by the strong law of large numbers, for every $v \in \Omega$ we have $G(u, v)=\int F^{v} d x$ for $\mu$-a.e. $u \in \Omega$. In particular, $G^{v}$ is $\mu$-a.e. constant for every $v \in \Omega$ since $G^{v}(u)=\int F^{v} d x=G^{v}\left(u^{\prime}\right)$ for $\mu$-a.e. $u, u^{\prime} \in \Omega$. Also, $G_{u}(v)$ is $\mu$-a.e. constant for every $u \in \Omega$ since it is the lim sup of $\mu$-a.e. constant functions $\frac{1}{n}\left[F_{u_{1}}(v)+\ldots+F_{u_{n}}(v)\right]$.

Put $E=\left\{\langle u, v\rangle \in \Omega^{2}: G(u, v) \leq a\right\}$. Since every vertical and horizontal section of $G$ is $\mu$-a.e. constant, it follows that every vertical and horizontal section of $E$ is either null or of full measure. Let us define $A_{1}=$ $\left\{u \in \Omega: \mu\left(E_{u}\right)=1\right\}, A_{2}=\left\{u \in \Omega: \mu\left(E_{u}\right)=0\right\}, B_{1}=\left\{v \in \Omega: \mu\left(E^{v}\right)=1\right\}$, and $B_{2}=\left\{v \in \Omega: \mu\left(E^{v}\right)=0\right\}$. By [6, 254 L Theorem, p. 249], we have $\bar{\mu}\left(U^{\omega}\right)=\bar{\mu}\left(V^{\omega}\right)=1$. If $u \in U^{\omega}$, then $G(u, v) \leq a$ for $\mu$-a.e. $v$; that is, $\mu\left(E_{u}\right)=1$. Similarly, if $u \in V^{\omega}$ then $\mu\left(E_{u}\right)=0$. Therefore $U^{\omega} \subset A_{1}$ and $V^{\omega} \subset A_{2}$ and thus $\bar{\mu}\left(A_{1}\right)=\bar{\mu}\left(A_{2}\right)=1$.

Suppose $\bar{\mu}\left(B_{2}\right)>0$. Then $E \cap\left(A_{1} \times B_{2}\right)$ is a $0-1$ set in $A_{1} \times B_{2}$ and $\left(A_{2} \times B_{1}\right) \backslash E$ is a $0-1$ set in $A_{2} \times B_{1}$. On the other hand, if $\bar{\mu}\left(B_{1}\right)>0$, then $\left(A_{2} \times B_{1}\right) \backslash E$ is a $0-1$ set in $A_{2} \times B_{1}$ and $E \cap\left(A_{1} \times B_{2}\right)$ is a $0-1$ set in $A_{1} \times B_{2}$. We deduce that the statement of $E_{\mathcal{N}}$ is true apart from the 
fact that the sets $A_{i}$ and $B_{i}$ are in $\Omega$ instead of $[0,1]$. However, the measure spaces $\Omega$ and $[0,1]$ are isomorphic, therefore we can find sets in $[0,1]$ with the same properties. Thus $E_{\mathcal{N}}$ holds, which completes the proof.

4. The category case. In this section we will prove that the category analogue of Theorem 7 is true with one obvious modification: the integral conditions (FP) and (SFP) have no meaning in this case.

Recall that $\mathcal{B}_{\mathcal{M}}$ coincides with the $\sigma$-algebra of sets $H \subset \mathbb{R}$ having the Baire property, and that (SAP) is equivalent to the following statement: if $A \subset[0,1]^{2}$ is such that $A_{x}$ and $A^{y}$ have the Baire property for every $x, y \in[0,1]$ then there is a set $B \subset[0,1]^{2}$ having the Baire property such that $(A \triangle B)_{x} \in \mathcal{M}$ and $(A \triangle B)^{y} \in \mathcal{M}$ for every $x, y \in[0,1]$.

Notice also that the chart below implies that all the properties considered there are independent of the axioms of ZFC. This is the case, since the relations $\operatorname{add}(\mathcal{M})=\operatorname{cov}(\mathcal{M})=\operatorname{non}(\mathcal{M})=2^{\omega}$ and $\operatorname{shr}(\mathcal{M})<\operatorname{cov}(\mathcal{M})$ are both consistent with ZFC: the first is a consequence of Martin's Axiom, while the second holds in the Cohen model. (For the proof of $\operatorname{shr}(\mathcal{M})<\operatorname{cov}(\mathcal{M})$ see [13] or [11].)

THEOREM 8. The following statements are equivalent to each other:

(i) $\mathcal{M}$ has property (SAP).

(ii) $\mathcal{M}$ has property (BAP).

(iii) $\mathcal{M}$ has property $\left(\mathrm{SKU}^{*}\right)$.

(iv) $E_{\mathcal{M}}$ is false.

In particular, for $\mathcal{I}=\mathcal{M}$ we have the following relations:

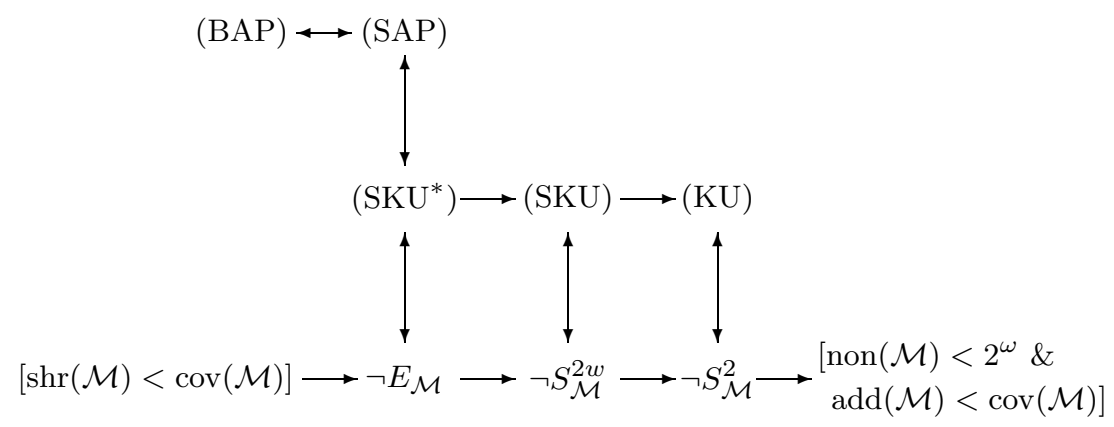

In the proof of the theorem we will need the following lemma.

Lemma 9. If $A \subset[0,1]^{2}$ is Borel then the set $\left\{y \in[0,1]: A^{y} \notin \mathcal{M}\right\}$ has the Baire property.

Proof. There is an open set $G \subset[0,1]^{2}$ such that $M=G \triangle A$ is meager. Then the set $\left\{y \in[0,1]: G^{y} \notin \mathcal{M}\right\}=\left\{y \in[0,1]: G^{y} \neq \emptyset\right\}$ is open, and the 
set $\left\{y \in[0,1]: M^{y} \notin \mathcal{M}\right\}$ is meager by the Kuratowski-Ulam theorem. Since $\left\{y \in[0,1]: A^{y} \notin \mathcal{M}\right\} \triangle\left\{y \in[0,1]: G^{y} \notin \mathcal{M}\right\} \subset\left\{y \in[0,1]: M^{y} \notin \mathcal{M}\right\}$, it follows that $\left\{y \in[0,1]: A^{y} \notin \mathcal{M}\right\}$ has the Baire property.

Proof of Theorem 8. The implications in the chart follow from the main part of the theorem, Theorem 1, and Corollary 3.

(i) $\Leftrightarrow($ ii): This follows from Fact 5 .

(ii) $\Rightarrow$ (iii): This follows from Lemma 9 and Proposition 6 .

(iii) $\Rightarrow$ (ii): Suppose that the sections of a set $A \subset[0,1]^{2}$ have the Baire property. It is clear from (SKU*) that for every interval $J$ the set $H_{J}=$ $\left\{y \in[0,1]: J \backslash A^{y} \in \mathcal{M}\right\}$ has the Baire property. Let

$$
E=\bigcup\left\{J \times H_{J}: J=(p, q) \subset[0,1] \text { for some rational numbers } p<q\right\} .
$$

Then $E$ has the Baire property, and it is easy to see that for $F=E \triangle A$ we have $F^{y}=E^{y} \triangle A^{y} \in \mathcal{M}$ for every $y \in[0,1]$. It is also clear that $F_{x}=E_{x} \triangle A_{x}$ has the Baire property for every $x \in[0,1]$. Since $\mathcal{M}$ has property $\left(\mathrm{SKU}^{*}\right)$, by Theorem 1 it also has property (KU) and so $U:=$ $\left\{x: F_{x} \notin \mathcal{M}\right\} \in \mathcal{M}$. Let $H=E \backslash(U \times[0,1])$. Then $H$ has the Baire property and we have $(A \triangle H)_{x} \in \mathcal{M}$ and $(A \triangle H)^{y} \in \mathcal{M}$ for every $x, y \in[0,1]$.

(iii) $\Rightarrow($ iv): This was proved in Theorem 1 .

(iv) $\Rightarrow$ (iii): We prove that if $\left(\mathrm{SKU}^{*}\right)$ is false then $E_{\mathcal{M}}$ is true. We will work in $\mathbb{R}$ instead of $[0,1]$.

Suppose that there exists a set $A \subset \mathbb{R}^{2}$ such that $A_{x}$ and $A^{y}$ have the Baire property for every $x, y \in \mathbb{R}$, and $H=\left\{y \in \mathbb{R}: A^{y} \notin \mathcal{M}\right\} \notin \mathcal{B}_{\mathcal{M}}$. By Banach's theorem, there exists a nonempty open set $G \subset \mathbb{R}$ such that $H \backslash G \in \mathcal{M}$ and $H$ is of second category in every nonempty open subset of $G$. (See $\left[15, \S 10, \mathrm{~V}\right.$, pp. 83-85].) Since $H \notin \mathcal{B}_{\mathcal{M}}$, the set $G \backslash H$ is not meager, and thus we can select an open interval $I \subset G$ such that $G \backslash H$ is of second category in every nonempty open subinterval of $I$. We conclude that both $H$ and $\mathbb{R} \backslash H$ are of second category in every nonempty open subinterval of $I$. Let $\phi$ be a homeomorphism from $I$ onto $\mathbb{R}$, and put

$$
C=\{\langle x, \phi(y)\rangle:\langle x, y\rangle \in A \cap(\mathbb{R} \times I)\} .
$$

Then $C_{x}$ and $C^{y}$ have the Baire property for every $x, y \in \mathbb{R}$, and the set $K=\left\{y \in \mathbb{R}: C^{y} \notin \mathcal{M}\right\}$ has the property that both $K$ and $\mathbb{R} \backslash K$ are of second category in every subinterval of $\mathbb{R}$. Let

$$
D=\{\langle x+r, y\rangle:\langle x, y\rangle \in C \& r \in \mathbb{Q}\} .
$$

Then $D^{y} \in \mathcal{M}$ if $y \notin K$ and $\mathbb{R} \backslash D^{y} \in \mathcal{M}$ if $y \in K$. Also, the set $D_{x}=\bigcup_{r \in \mathbb{Q}} C_{x-r}$ has the Baire property for every $x \in \mathbb{R}$. Let us put $U=$ $\left\{x \in \mathbb{R}: \mathbb{R} \backslash D_{x} \in \mathcal{M}\right\}$. We shall distinguish between two cases. 
Case I: $\mathbb{R} \backslash U \in \mathcal{M}$. Then the set $D \cap[U \times(\mathbb{R} \backslash K)]$ is a $0-1$ set in $U \times(\mathbb{R} \backslash K)$, and $(\mathbb{R} \backslash U) \times K$ is a $0-1$ set in $(\mathbb{R} \backslash U) \times K$. That is, $E_{\mathcal{M}}$ holds in this case.

Case II: $\mathbb{R} \backslash U \notin \mathcal{M}$. If $x \in \mathbb{R} \backslash U$ then $\mathbb{R} \backslash D_{x} \notin \mathcal{M}$ and, as $D_{x}$ has the Baire property, there exists an open interval $J$ with rational endpoints such that $D_{x} \cap J \in \mathcal{M}$. Since $\mathbb{R} \backslash U \notin \mathcal{M}$, it follows that we can fix an open interval $J$ with rational endpoints such that the set $V=\left\{x \in \mathbb{R}: D_{x} \cap J \in \mathcal{M}\right\}$ is of second category. Let $\psi$ be a homeomorphism from $J$ onto $\mathbb{R}$, and put

$$
E=\{\langle x, \psi(y)\rangle:\langle x, y\rangle \in D \cap(\mathbb{R} \times J)\} .
$$

Then $E^{y} \in \mathcal{M}$ if $y \notin \psi[K \cap J]$ and $\mathbb{R} \backslash E^{y} \in \mathcal{M}$ if $y \in \psi[K \cap J]$. Also, the set $W=\left\{x \in \mathbb{R}: E_{x} \in \mathcal{M}\right\}$ is of second category. Now we define

$$
F=\{\langle x, y+r\rangle:\langle x, y\rangle \in E \& r \in \mathbb{Q}\} .
$$

Then either $F^{y} \in \mathcal{M}$ or $\mathbb{R} \backslash F^{y} \in \mathcal{M}$ for every $y$. If $Y=\left\{y: \mathbb{R} \backslash F^{y} \in \mathcal{M}\right\}$ then $Y \notin \mathcal{M}$, as $\psi[K \cap J] \subset Y$. Also, we have $F_{x} \in \mathcal{M}$ if $x \in W$, and $\mathbb{R} \backslash F_{x} \in \mathcal{M}$ if $x \notin W$. Thus $(W \times Y) \backslash F$ is a 0 - 1 set in $W \times Y$ and $[(\mathbb{R} \backslash W) \times(\mathbb{R} \backslash Y)] \cap F$ is a 0 -1 set in $(\mathbb{R} \backslash W) \times(\mathbb{R} \backslash Y)$. Therefore, $E_{\mathcal{M}}$ holds in this case as well.

5. More on condition $E_{\mathcal{I}}$. The definition of $E_{\mathcal{I}}$ does not involve the topology of the space $X$; it is meaningful for any ideal of subsets of a set $X$. We begin with an equivalent form of $E_{\mathcal{I}}$. We shall use the notation $A^{\mathrm{c}}=X \backslash A$.

Proposition 10. For every ideal $\mathcal{I}$ the statement $E_{\mathcal{I}}$ is equivalent to the following:

$E_{\mathcal{I}}^{*}$ : $\quad$ Either there exists a set $A \subset X$ such that $A, A^{\mathrm{c}} \notin \mathcal{I}$ and there is a 0-1 set in $A \times A^{\mathrm{c}}$, or there exists a set $A \subset X$ such that there are 0-1 sets both in $A \times A$ and in $A^{\mathrm{c}} \times A^{\mathrm{c}}$.

Proof. Suppose $E_{\mathcal{I}}^{*}$. If $H$ is a $0-1$ set in $A \times A^{\mathrm{c}}$ where $A, A^{\mathrm{c}} \notin \mathcal{I}$, then $E_{\mathcal{I}}$ is satisfied with $B=A^{\mathrm{c}}$. If there are 0-1 sets both in $A \times A$ and in $A^{\mathrm{c}} \times A^{\mathrm{c}}$ then we may assume $A \notin \mathcal{I}$, since otherwise we replace $A$ by $A^{\mathrm{c}}$. Then $E_{\mathcal{I}}$ is satisfied with $B=A$.

Next suppose $E_{\mathcal{I}}$. Suppose that $A, B \notin \mathcal{I}, H$ is a 0 - 1 set in $A \times B$ and $K$ is a $0-1$ set in $A^{\mathrm{c}} \times B^{\mathrm{c}}$. If $A \backslash B \in \mathcal{I}$ and $B \backslash A \in \mathcal{I}$ then there are 0-1 sets both in $A \times A$ and in $A^{\mathrm{c}} \times A^{\mathrm{c}}$, and then $E_{\mathcal{I}}^{*}$ holds.

Therefore we may assume that at least one of the sets $A \backslash B$ and $B \backslash A$ does not belong to $\mathcal{I}$. By symmetry, we may assume that $D:=A \backslash B \notin \mathcal{I}$. We also have $D^{\mathrm{c}} \notin \mathcal{I}$, since $D^{\mathrm{c}} \supset B$. We prove that there is a $0-1$ set in $D \times D^{\mathrm{c}}$. Indeed, we have $D^{\mathrm{c}}=A^{\mathrm{c}} \cup B$, and thus

$$
D \times D^{\mathrm{c}}=\left(D \times A^{\mathrm{c}}\right) \cup(D \times B) .
$$


Since $D \times A^{\mathrm{c}} \subset B^{\mathrm{c}} \times A^{\mathrm{c}}$ and $D \times B \subset A \times B$, it follows that there are 0-1 sets both in $D \times A^{\mathrm{c}}$ and in $D \times B$, and then there is also one in $D \times D^{\mathrm{c}}$.

In what follows we shall assume that $X$ is an Abelian group and $\mathcal{I}$ is a translation invariant ideal in $X$. The paper [17] investigated the logical connections between several statements including $S_{\mathcal{I}}^{2}$ and $S_{\mathcal{I}}^{2 w}$ and the following three conditions.

$S_{\mathcal{I}}^{1}$ : $\quad$ There exists a set $A \subset X$ such that $A \notin \mathcal{I}, X \backslash A \notin \mathcal{I}$, and $(A+h) \backslash A \in$ $\mathcal{I}$ for every $h \in X$.

$S_{\mathcal{I}}^{1 s}: \quad$ There exists an $f: X \rightarrow X$ such that $\{x \in X: f(x+h)-f(x) \neq 0\} \in \mathcal{I}$ for every $h \in X$, and $f^{-1}(\{y\}) \in \mathcal{I}$ for every $y \in X$.

$D_{\mathcal{I}}$ : There exists a partition $\{A, B\}$ of $X$ such that $\operatorname{cov}(\mathcal{I} \mid A) \leq \operatorname{non}(\mathcal{I} \mid B)$ and $\operatorname{cov}(\mathcal{I} \mid B) \leq \operatorname{non}(\mathcal{I} \mid A)$.

In [17, Theorem 2] it was shown that if $\mathcal{I}$ is a $\sigma$-ideal and $|X|$ is less than the first (2-valued) measurable cardinal then the following implications hold.

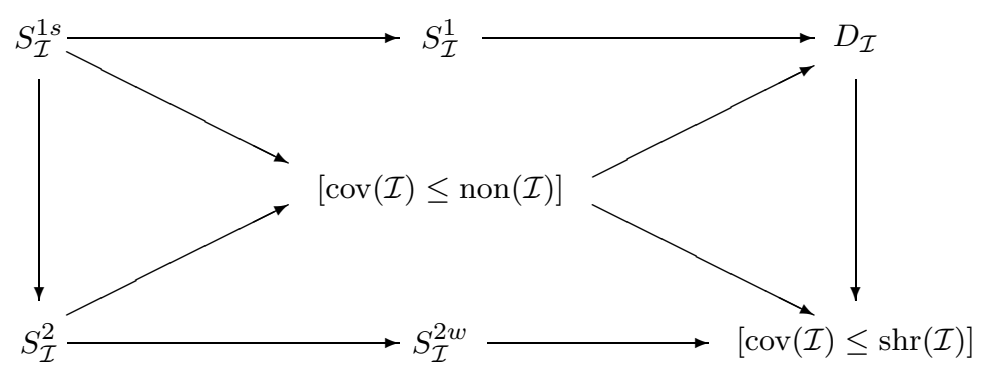

Diagram 1

In the next theorem we try to locate the position of $E_{\mathcal{I}}$ in this diagram. As we shall see, it must be somewhere in the middle column.

Theorem 11. Let $\mathcal{I}$ be an invariant ideal in the Abelian group $X$. Then we have the following implications:

$$
\left[S_{\mathcal{I}}^{1} \text { or } S_{\mathcal{I}}^{2 w}\right] \Rightarrow E_{\mathcal{I}} \Rightarrow\left[S_{\mathcal{I}}^{1} \text { or }(\operatorname{cov}(\mathcal{I}) \leq \operatorname{non}(\mathcal{I})) \text { or } S_{\mathcal{I}}^{2 w}\right] .
$$

In the proof of the theorem we will use the following lemma.

Lemma 12. Let $\mathcal{I}$ be an invariant ideal in an Abelian group $X$. If there is a partition $\{A, B\}$ of $X$ such that $\operatorname{cov}(\mathcal{I} \mid A) \leq \operatorname{non}(\mathcal{I} \mid A)$ and $\operatorname{cov}(\mathcal{I} \mid B) \leq$ $\operatorname{non}(\mathcal{I} \mid B)$ then $\operatorname{cov}(\mathcal{I}) \leq \operatorname{non}(\mathcal{I})$.

Proof. If $\operatorname{non}(\mathcal{I} \mid A)=\operatorname{non}(\mathcal{I} \mid B)=\kappa$ then $\operatorname{non}(\mathcal{I})=\kappa$ and

$$
\operatorname{cov}(\mathcal{I})=\max \{\operatorname{cov}(\mathcal{I} \mid A), \operatorname{cov}(\mathcal{I} \mid B)\} \leq \kappa .
$$


Therefore we may assume that $\operatorname{non}(\mathcal{I} \mid A) \neq \operatorname{non}(\mathcal{I} \mid B)$. By symmetry we may suppose $\operatorname{non}(\mathcal{I} \mid A)<\operatorname{non}(\mathcal{I} \mid B)$. Let $\operatorname{non}(\mathcal{I} \mid A)=\lambda$ and let $H \subset A$ be a set with $|H|=\lambda$ and $H \notin \mathcal{I}$. Then for every $x \in X$ we have $H+x \notin \mathcal{I}$ and $|H+x|=\lambda<\operatorname{non}(\mathcal{I} \mid B)$. Thus $H+x$ cannot be a subset of $B$. Therefore $(H+x) \cap A \neq \emptyset$ for every $x$. This implies that for every $x$ there is an $h \in H$ such that $h+x \in A$, that is, $x \in A-h$. Thus $X=\bigcup_{h \in H}(A-h)$.

Since $A-h$ can be covered by $\operatorname{cov}(\mathcal{I} \mid A) \leq \lambda$ elements of $\mathcal{I}$ and $|H|=\lambda$, it follows that $X$ can also be covered by $\lambda$ elements of $\mathcal{I}$. In other words, $\operatorname{cov}(\mathcal{I}) \leq \lambda=\operatorname{non}(\mathcal{I})$

Proof of Theorem 11. $S_{\mathcal{I}}^{2 w} \Rightarrow E_{\mathcal{I}}$ was proved in Theorem 1.

$S_{\mathcal{I}}^{1} \Rightarrow E_{\mathcal{I}}$ : Suppose $S_{\mathcal{I}}^{1}$, and let $A \subset X$ be a set such that $A \notin \mathcal{I}$, $X \backslash A \notin \mathcal{I}$, and $(A+h) \backslash A \in \mathcal{I}$ for every $h \in X$. We claim that there is a $0-1$ set in $A \times A^{\mathrm{c}}$. Indeed, let $H=\left\{\langle x, y\rangle \in A \times A^{\mathrm{c}}: y \notin(A-x) \backslash A\right\}$. For every $x \in A$ we have $A^{\mathrm{c}} \backslash H_{x}=(A-x) \backslash A \in \mathcal{I}$. If $y \in A^{\mathrm{c}}$ then we have

$$
\begin{aligned}
\langle x, y\rangle \in H & \Rightarrow y \notin A-x \Rightarrow x \notin A-y \\
& \Rightarrow x \in A \backslash(A-y)=[(A+y) \backslash A]-y \in \mathcal{I},
\end{aligned}
$$

and thus $H^{y} \in \mathcal{I}$ for every $y \in A^{\mathrm{c}}$. Therefore, $H$ is a $0-1$ set in $A \times A^{\mathrm{c}}$.

Now we turn to the proof of the second implication of Theorem 11. Suppose that $E_{\mathcal{I}}$ holds. By Proposition 10, one of the following statements is true: (i) there exists a set $A \subset X$ such that $A, A^{\mathrm{c}} \notin \mathcal{I}$ and there is a $0-1$ set in $A \times A^{\mathrm{c}}$, or (ii) there exists a set $A \subset X$ such that there are 0-1 sets both in $A \times A$ and in $A^{\mathrm{c}} \times A^{\mathrm{c}}$.

Suppose (ii). If $A^{\mathrm{c}} \in \mathcal{I}$ then there is a $0-1$ set in $X \times X$, and thus $S_{\mathcal{I}}^{2 w}$ (even $S_{\mathcal{I}}^{2}$ ) holds. We have the same conclusion if $A \in \mathcal{I}$. If $A, X \backslash A \notin \mathcal{I}$ then, by Lemma 2 , we obtain $\operatorname{cov}(\mathcal{I} \mid A) \leq \operatorname{non}(\mathcal{I} \mid A)$ and $\operatorname{cov}\left(\mathcal{I} \mid A^{\mathrm{c}}\right) \leq \operatorname{non}\left(\mathcal{I} \mid A^{\mathrm{c}}\right)$. Therefore, by Lemma 12 , we get $\operatorname{cov}(\mathcal{I}) \leq \operatorname{non}(\mathcal{I})$.

Next suppose (i). Then there exists a set $A \subset X$ such that $A, A^{\mathrm{c}} \notin \mathcal{I}$, and there is a $0-1$ set in $A \times A^{\mathrm{c}}$. If $(A+h) \backslash A \in \mathcal{I}$ for every $h \in X$ then we have $S_{\mathcal{I}}^{1}$. Therefore we may assume that there is an $h \in X$ such that $B:=(A+h) \backslash A=(A+h) \cap A^{\mathrm{c}} \notin \mathcal{I}$. Since

$$
B \times X \subset\left[(A+h) \times A^{\mathrm{c}}\right] \cup\left[A^{\mathrm{c}} \times A\right]
$$

and there are $0-1$ sets in both $(A+h) \times A^{\mathrm{c}}$ and $A^{\mathrm{c}} \times A$, it follows that there is a $0-1$ set in $B \times X$, and thus $S_{\mathcal{I}}^{2 w}$ holds.

We conclude with another simple observation.

Proposition 13. For every invariant ideal $\mathcal{I}$ on an Abelian group $X$ we have $S_{\mathcal{I}}^{2 w} \Rightarrow D_{\mathcal{I}}$ and $E_{\mathcal{I}} \Rightarrow D_{\mathcal{I}}$.

Proof. Suppose that $S_{\mathcal{I}}^{2 w}$ holds. Then there exists a set $A \subset X$ such that $A \notin \mathcal{I}$, and there is a $0-1$ set in $A \times X$. If $A^{\mathrm{c}} \in \mathcal{I}$ then $S_{\mathcal{I}}^{2}$ holds, which implies $D_{\mathcal{I}}$ by Diagram 1 . Therefore we may assume $A^{\mathrm{c}} \notin \mathcal{I}$. 
Since there is a $0-1$ set in $A \times X$, there is also one in $A \times A^{\mathrm{c}}$ and hence in $A^{\mathrm{c}} \times A$ as well. Then, by Lemma 2 , it follows that $\operatorname{cov}(\mathcal{I} \mid A) \leq \operatorname{non}\left(\mathcal{I} \mid A^{\mathrm{c}}\right)$ and $\operatorname{cov}\left(\mathcal{I} \mid A^{\mathrm{c}}\right) \leq \operatorname{non}(\mathcal{I} \mid A)$; that is, $D_{\mathcal{I}}$ holds.

Now each of the statements $S_{\mathcal{I}}^{1}, \operatorname{cov}(\mathcal{I}) \leq \operatorname{non}(\mathcal{I})$, and $S_{\mathcal{I}}^{2 w}$ implies $D_{\mathcal{I}}$. (For the first two of these implications we again refer to Diagram 1.) Therefore, by Theorem 11, we have $E_{\mathcal{I}} \Rightarrow D_{\mathcal{I}}$.

We remark that the implication $S_{\mathcal{I}}^{2 w} \Rightarrow D_{\mathcal{I}}$ was not noted in [17]. It would be interesting to decide whether or not there are other implications that can be inserted into Diagram 1.

\section{References}

[1] A. Cauchy, Mémoire sur les intégrales définies, Acad. Sci. Paris Mém. 1 (1827). Reprinted in: Oeuvres complètes d'Augustin Cauchy, Gauthier-Villars, 1882, Vol. I, 319-506.

[2] K. Ciesielski, Set Theory for the Working Mathematician, London Math. Soc. Stud. Texts 39, Cambridge Univ. Press, 1997.

[3] C. de la Vallée-Poussin, Réduction des intégrales doubles de Lebesgue: Application à la définition des fonctions analytiques, Bull. Cl. Sci. Acad. Roy. Belgique 12 (1910), 768-798.

[4] P. du Bois-Reymond, Über das Doppelintegral, J. Reine Angew. Math. 94 (1883), 273-290.

[5] C. Freiling, Axioms of symmetry: throwing darts at the real number line, J. Symbolic Logic 51 (1986), 190-200.

[6] D. H. Fremlin: Measure Theory, Vol. 2, Torres Fremlin, Colchester, 2001.

[7] H. Friedman, A consistent Fubini-Tonelli theorem for non-measurable functions, Illinois J. Math. 24 (1980), 390-395.

[8] G. Fubini, Sugli integrali multipli, Atti Reale Accad. Lincei Rend. (5) 16 (1907), 608-614.

[9] T. Hawkins, Lebesgue's Theory of Integration, 2nd ed., Chelsea, 1975.

[10] E. W. Hobson, On some fundamental properties of Lebesgue integrals in a twodimensional domain, Proc. London Math. Soc. 8 (1910), 22-39.

[11] M. Kada and Y. Yuasa, Cardinal invariants about shrinkability of unbounded sets, Topology Appl. 74 (1996), 215-223.

[12] A. S. Kechris, Classical Descriptive Set Theory, Grad. Texts in Math. 156, Springer, 1995.

[13] P. Komjáth, Some remarks on second category sets, Colloq. Math. 66 (1993), 57-62.

[14] K. Kunen, Random and Cohen reals, in: Handbook of Set-Theoretic Topology, North-Holland, 1984, 887-911.

[15] K. Kuratowski, Topology, Vol. I, Academic Press, 1966.

[16] M. Laczkovich, Fubini's theorem and Sierpiński's set, preprint, 1985.

[17] —, Two constructions of Sierpinski and some cardinal invariants of ideals, Real Anal. Exchange 24 (1998-99), 663-676.

[18] M. Laczkovich and A. W. Miller, Measurability of functions with approximately continuous vertical sections and measurable horizontal sections, Colloq. Math. 69 (1995), 299-308. 
[19] H. Lebesgue, Sur les fonctions représentables analytiquement, J. Math. Pures Appl. 1 (1905), 139-215.

[20] L. Lichtenstein, Sur une condition d'intégrabilité des binômes différentielles et sur le théorème fondamentale de la théorie des fonctions analytiques, Prace Mat.-Fiz. 22 (1911), 1-17 (in Polish).

[21] I. Recław and P. Zakrzewski, Strong Fubini properties of ideals, Fund. Math. 159 (1999), 135-152.

[22] W. Sierpiński, Sur les rapports entre l'existence des intégrales $\int_{0}^{1} f(x, y) d x$, $\int_{0}^{1} f(x, y) d y$ et $\int_{0}^{1} d x \int_{0}^{1} f(x, y) d y$, ibid. 1 (1920), 142-147. Reprinted in: Oeuvres Choisies, PWN, 1975, Vol. II, 341-345.

Department of Mathematics

West Virginia University

Morgantown, WV 26506-6310, U.S.A.

E-mail: K_Cies@math.wvu.edu

Web: http://www.math.wvu.edu/ ${ }^{\sim}$ kcies
Department of Analysis Eötvös Loránd University Pázmány Péter sétány 1/C 1117 Budapest, Hungary E-mail: laczko@renyi.hu

Department of Mathematics University College London WC1E 6BT London, England E-mail: laczk@math.ucl.ac.uk 